\title{
Analysis of Gait Changes Caused by Simulated Left Knee Disorder
}

\author{
Takuya Ogawa \\ Osaka University \\ 8-1 Mihogaoka, Ibaraki \\ Osaka, Japan
}

\author{
Hirotake Yamazoe \\ Ritsumeikan University/Osaka \\ University 1-1-1 Nojihigashi, \\ Kusatsu \\ Shiga, Japan \\ yamazoe@fc.ritsumei.ac.jp \\ Yasushi Yagi \\ Osaka University \\ 8-1 Mihogaoka, lbaraki \\ Osaka, Japan
}

\author{
Ikuhisa Mitsugami \\ Osaka University \\ 8-1 Mihogaoka, lbaraki \\ Osaka, Japan
}

\begin{abstract}
In this paper, we analyze the gait changes by simulating left knee disorders in subjects. Our aim is a method that estimates the presence or the absence of leg disorders and the disordered parts from the image sequences of a subject's walking. However, gait can be changed not only by physical disorders but also such factors as neural disorders or aging. Thus we simulate the physical disorders using a knee brace. Healthy subjects wore knee braces. We compared the normal and simulated disordered walking (while wearing the knee brace) to analyze what changes occur in their gait due to physical disorders. We also analyzed whether the changes are common to all subjects. Analysis results show that the changes common to all subjects are caused by the simulated left knee disorders.
\end{abstract}

\section{Categories and Subject Descriptors}

J.3 [Computer Applications]: Life and Medical Sciences,Health

\section{General Terms}

Human Factors

\section{INTRODUCTION}

Gait changes can be caused by such factors as osteoarthritis of the knee or hip, Alzheimer's disease, and injuries to the inner ear, etc. Many researches have addressed the effects of such diseases on gait $[1,5,4]$.

In this paper, we focus on knee osteoarthritis and analyze the effects on gait caused by knee contractures (limitations of the joint Affs range of motion), which are one major symptom of knee osteoarthritis. Some researchers have analyzed the gait changes of knee osteoarthritis and the effects of knee contractures on their gait $[3,2]$. In their previous work that analyzed the effects on gait by knee contractures, they simulated knee contractures using a knee brace, which was worn by healthy participants, and analyzed the gaits with and without it. In this paper, we also simulated the knee contracture using a knee brace and analyzed the gait changes due to knee contracture. Unlike the previous work [3, 2], which focused only on the lower limb motions, we measured and analyzed the whole-body motion of the participants and investigated whether the gait changes that are caused by the simulated knee disorders are common to all the subjects.

\section{EXPERIMENT}

\subsection{Walking Data Capturing}

First, we describe how we captured the walking data. Fig. 1 shows the brace for simulating a knee disorder, and Fig. 2 shows the experimental environment, where we placed six cameras for a motion capture system (Bonita 10, Vicon Motion Systems Ltd., UK) that covered the area shown in Fig. 2. Approximately four steps (two walking cycles) can be captured in the capturing area.

We conducted walking-data-capture with 13 males First, they walked three laps without a knee brace, and these walking data are called normal walking. Next, the participants wore a knee brace and walked more than ten minutes. Here, our preliminary experiment showed that the gait is changed gradually after wearing the knee brace and it takes about five minutes to stabilize the changes. Thus, disordered walking is the walking data captured after the participants wore the knee brace for ten minutes. In the walking-data-capture, the participants followed a mobility scooter (Fig. 1, left), which ran at a constant speed, to remove the effects of walking speed changes.

From the obtained data, we calculated the 3D positions of the following 15 body parts (Fig. 3): head, neck, breast (bre), shoulders (lsh, rsh), elbows (lel, rel), wrists (lwr, rwr), waists (lwt, rwt), knees (lkn, rkn), and ankles (lan, ran). 

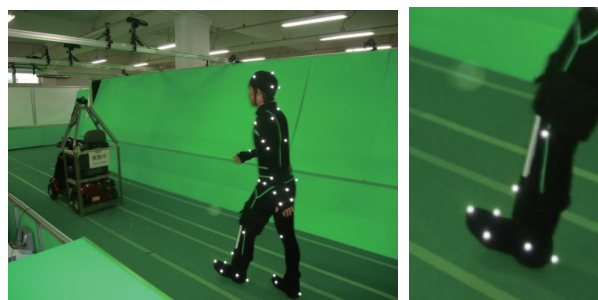

Figure 1: Experimental setup and knee brace

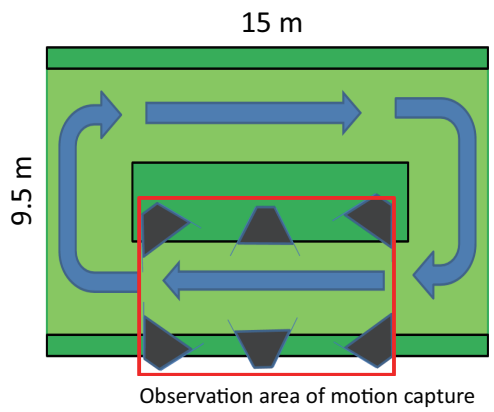

Figure 2: Experimental environment

\subsection{Preprocess of Walking Data}

Before analyzing the walking data, we performed the following preprocessing. In the analysis, we focused on the fact that walking is a periodic motion. Walking data during one walking cycle (two steps) include all the walking motions. Thus, we segmented the walking data into one bit of walking cycle data and analyzed every cycle of data. We defined the periods from the moment the left leg contacts the ground to the moment just before the next left leg contacts as the walking cycles.

A walking cycle is divided into four phases by each leg phase (stance/swing): double support 1, single support (left), double support 2, and single support (right) (Table 1).

Here, since the durations of the walking cycle and each nor-

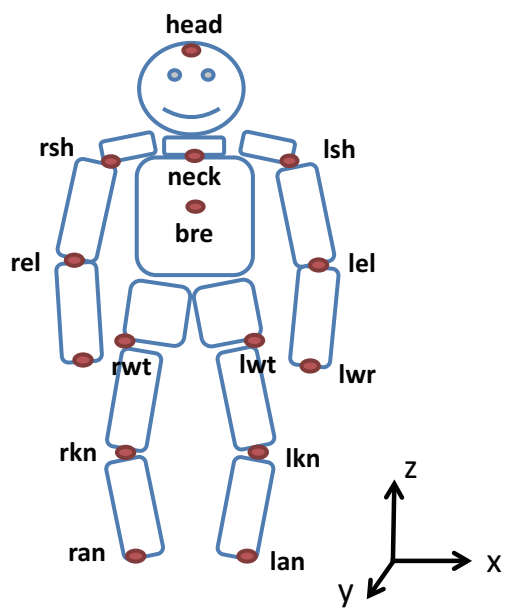

Figure 3: Points used for analysis
Table 1: Walking phases

\begin{tabular}{l|l}
\hline Double support 1 & Left leg is ahead of right leg \\
Single support (left) & Right leg is swing phase \\
Double support 2 & Right leg is ahead of left leg \\
Single support (right) & Left leg is swing phase \\
\hline
\end{tabular}

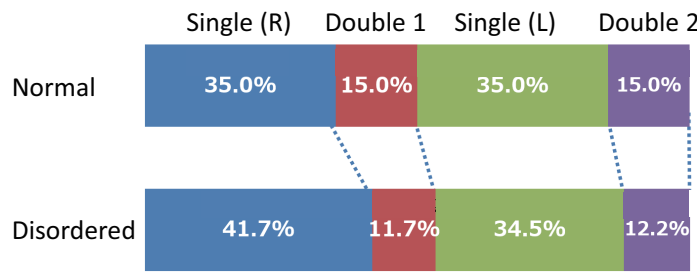

Figure 4: Comparison of phase durations

mal walking and disordered walking phase are different, we cannot directly compare the obtained motion data.

Instead, we performed phase registration between the normal walking and distortion walking data using Dynamic Programming (DP). After the registration, we compared both bits of data. To perform the phase registration, we converted the $3 \mathrm{D}$ positions of all the points to a relative coordinate system whose origin is the average of the waists (lwt and rwt).

\subsection{Phase Length Analysis}

We first analyzed the changes of each phase length.

From the DP-based phase registration results, we calculated each phase length in the normalized walking cycles. Fig. 4 shows the changes of the phase length.

Wearing knee braces prolong the duration of the single support (right) and shortens the durations of both double supports. On the other hand, we found no big changes in the duration of the single support (left).

\subsection{Pose Analysis}

Next, we show the pose analysis results in the comparison of the normal and disordered walking.

Figs. 5-13 show the averaged 3D positions of all the participants. The red and blue lines denote the averaged results of the normal and disordered walking. The dotted lines show the standard deviations of normal walking.

Between normal and disordered walking, the comparison results show various differences, which can be divided into three as follows.

\subsubsection{Differences in center parts}

From Figs. 5-8, the amplitudes of the left-right directions ( $\mathrm{x}$ axis) of the center parts (head, neck, bre, lsh, and rsh) increase in the disordered condition. In addition, the positions of the center parts move forward (y axis), meaning the upper body leans to the front. 

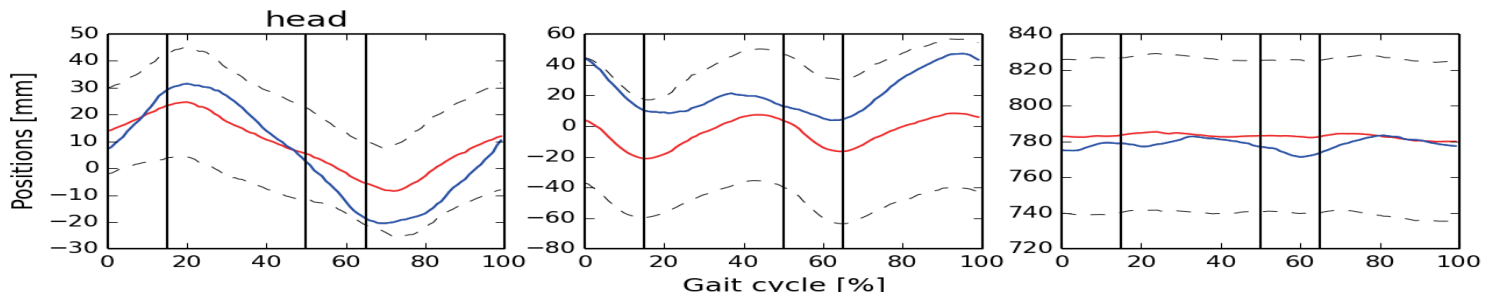

Figure 5: Comparison of head positions
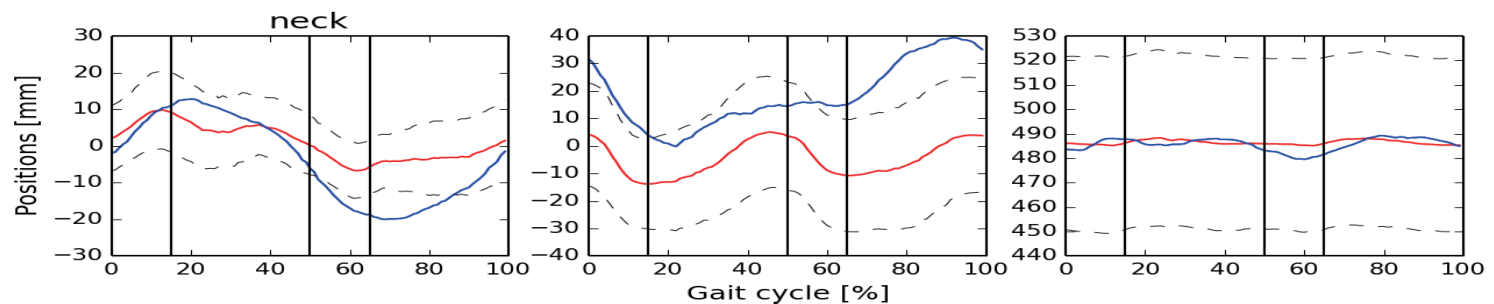

Figure 6: Comparison of neck positions
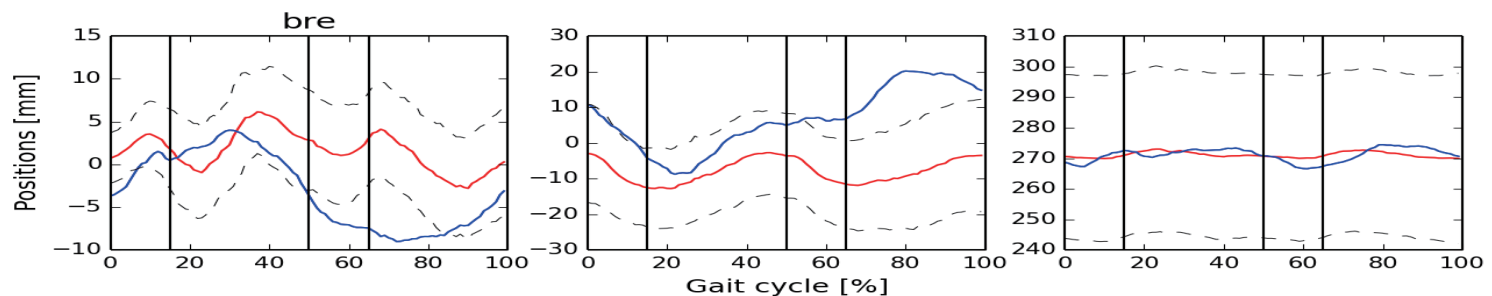

Figure 7: Comparison of breast positions

\subsubsection{Differences in upper limbs}

From Figs. 9-10, the right arm tends to lean to the right and left arm tends to lean to the left. In the y and $\mathrm{z}$ axes, there were no big differences between the normal and disordered walking.

\subsubsection{Differences in lower limbs}

From Figs. 11-13, the right knee and ankle tend to move to the right and the left knee and ankle tend to move to the left. Further, the left knee and ankle tend to move backward. In addition, the vertical motions of both waists decreased in the disordered walking. The right waist tends to move forward, and the left waist tends to move backward.

\section{CONCLUSION}

In this paper, we analyzed gait changes by simulating left knee disorders in subjects. Healthy subjects wore a knee brace, and we compared their normal and simulated disordered walking. Our analysis results showed that the changes shared by all subjects are caused by the simulated left knee disorders. Future works will analyze the effect of braces on gait and develop an estimation method of leg disorders from subject gaits.

\section{ACKNOWLEDGMENT}

This research was partly supported by the JST CREST "Behavior Understanding based on Intention-Gait Model" project.

\section{REFERENCES}

[1] J. R. Gage, D. Fabian, R. Hicks, and S. Tashman. Pre-and postoperative gait analysis in patients with spastic diplegia: a preliminary report. Journal of Pediatric Orthopaedics, 4(6):715-725, 1984.

[2] K. Harato, T. Nagura, H. Matsumoto, T. Otani, Y. Toyama, and Y. Suda. A gait analysis of simulated knee flexion contracture to elucidate knee-spine syndrome. Gait and Posture, 28(4):687-692, 2008.

[3] K. Harato, T. Nagura, H. Matsumoto, T. Otani, Y. Toyama, and Y. Suda. Knee flexion contracture will lead to mechanical overload in both limbs: A simulation study using gait analysis. The Knee, 15(6):467-472, 2008.

[4] C.-J. Lin, L.-Y. Guo, F.-C. Su, Y.-L. Chou, and R.-J. Cherng. Common abnormal kinetic patterns of the knee in gait in spastic diplegia of cerebral palsy. Gait \& posture, 11(3):224-232, 2000.

[5] S. Rose, P. DeLuca, R. Davis III, S. Ounpuu, and J. Gage. Kinematic and kinetic evaluation of the ankle after lengthening of the gastrocnemius fascia in children with cerebral palsy. Journal of Pediatric Orthopaedics, 13(6):727-732, 1993. 


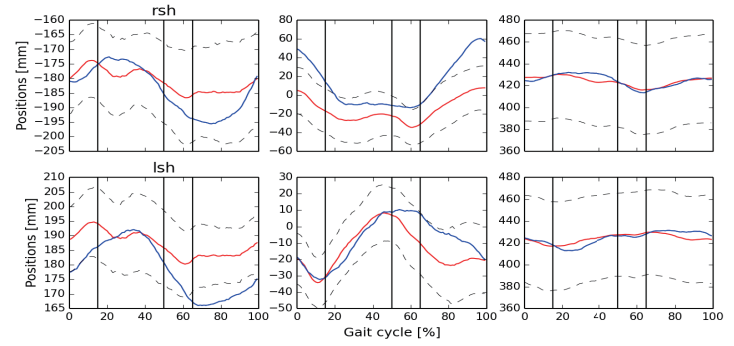

Figure 8: Comparison of shoulder positions

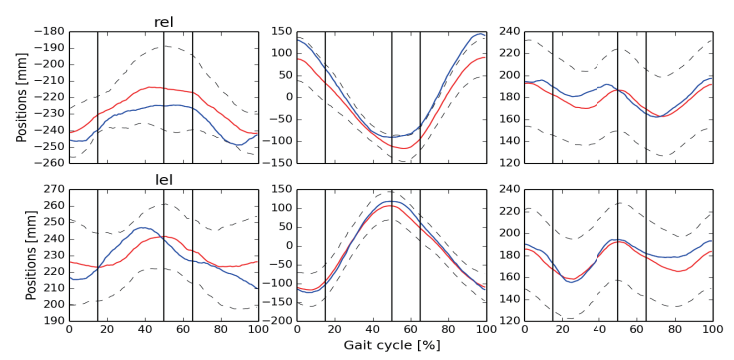

Figure 9: Comparison of elbow positions

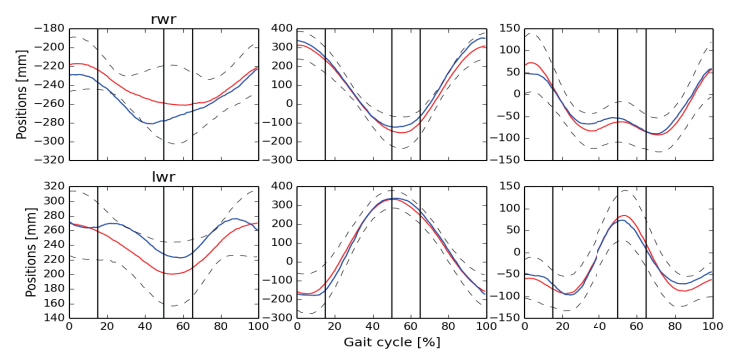

Figure 10: Comparison of wrist positions

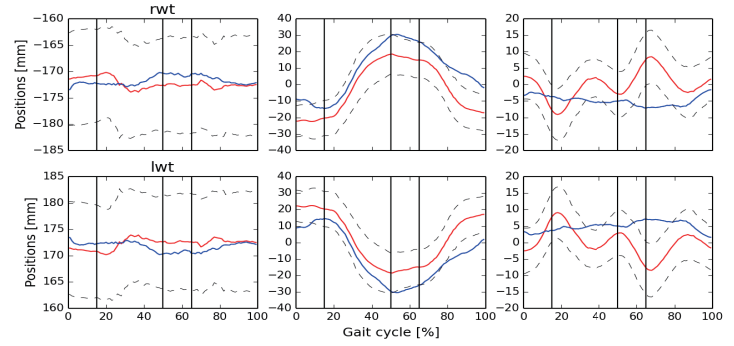

Figure 11: Comparison of waist positions

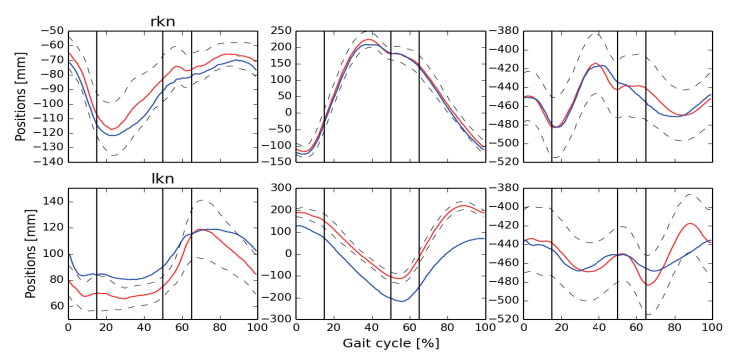

Figure 12: Comparison of knee positions

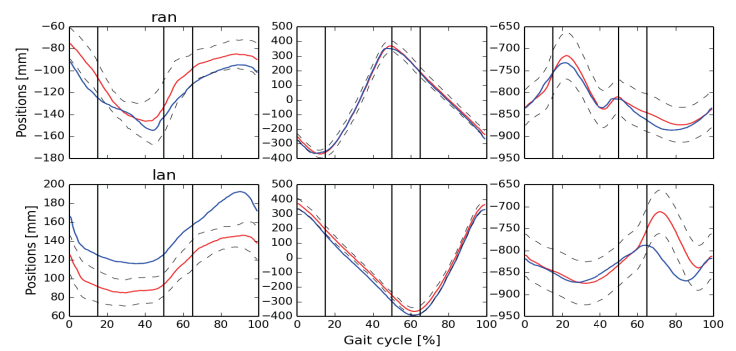

Figure 13: Comparison of ankle positions 